\title{
Priorities for representation: Task settings and object interaction both influence object memory
}

\author{
Clare Kirtley $^{1} \cdot$ Benjamin W. Tatler ${ }^{1}$
}

Published online: 3 September 2015

(C) Psychonomic Society, Inc. 2015

\begin{abstract}
Following an active task, the memory representations for used and unused objects are different. However, it is not clear whether these differences arise due to prioritizing objects that are task-relevant, objects that are physically interacted with, or a combination of the two factors. The present study allowed us to tease apart the relative importance of task-relevance and physical manipulation on object memory. A paradigm was designed in which objects were either necessary to complete a task (target), moved out of the way (obstructing, but interacted with), or simply present in the environment (background). Participants' eye movements were recorded with a portable tracker during the task, and they received a memory test on the objects after the task was completed. Results showed that manipulating an object is sufficient to change how information is extracted and retained from fixations, compared to background objects. Taskrelevance provides an additional influence: information is accumulated and retained differently for manipulated target objects than manipulated obstructing objects. These findings demonstrate that object memory is influenced both by whether we physically interact with an object, and the relevance of that object to our behavioral goals.
\end{abstract}

Keywords Action · Perception · Memory $\cdot$ Intention · Real-world

In everyday life, we perform actions in order to achieve a goal, informed by perceptual input about the current state of the

Clare Kirtley

clare.kirtley@abdn.ac.uk

1 School of Psychology, University of Aberdeen, Aberdeen AB24 2UB, UK environment. Despite this strong link between perception and action, it is only more recently that this has been represented in cognitive theories; in particular, those of grounded cognition. There are multiple iterations of these theories (see e.g., Wilson, 2002), but they all make the same underlying proposal: that perception and action are more than low-level input/output devices: instead, they are both dynamic and inter-related processes which allow us to navigate and work within our environment.

There is growing evidence that action influences perception. Laboratory-based tasks have demonstrated how preparing an action can affect the performance of visual search tasks: for example, Fagioli, Hommel, and Schubotz (2007) showed that, depending on the gesture prepared prior to search (either pointing or grasping), detection of an oddball target stimulus was facilitated if the gesture was compatible with the relevant feature of the target. Thus, preparing pointing actions facilitated the detection of location-defined stimuli, while preparing grasping actions assisted the detection of size-defined stimuli. Similar results were found in a change detection study (Symes, Tucker, Ellis, Vainio, and Ottoboni, 2008). By preparing a particular grip type (power or precision), changes made to stimuli compatible with the size of the grip aperture were spotted faster and more accurately than changes to the grip-incompatible objects. In both cases, it is apparent that the mere preparation of a particular gesture is enough to bias attention to those items in a scene containing some feature that makes them relevant, enhancing processing of such an item in preparation for a possible interaction in the future.

It is not only laboratory tasks that show effects of action. Studies which place participants into a real-world environment with real-world demands show a similarly close link between perceptual processes (usually visual behavior) and the responses required for the task. In an eye-tracking study of tea-making, it was found that the typical pattern of eye movements was to precede the hands to the object that was needed, and remain fixating the object in order to monitor any 
manipulations for as long as was required (Land, Mennie, and Rusted, 1999). Furthermore, it is the relevant items that receive the most attention; with a clear and significant division between relevant and irrelevant items in terms of the number of fixations they receive (Hayhoe, Shrivastava, Mruczec, and Pelz, 2003). Indeed, manual manipulation and gaze direction are co-located for the majority of time during many behaviors (Ballard, Hayhoe, Li, and Whitehead, 1992). In the course of our everyday lives, vision is thus constantly in the service of action, and it is the nature of the action which drives and directs vision.

While the majority of these studies demonstrate active vision in an on-line capacity, there are other studies which show that such effects are not only limited to situations in which the objects remain present: Memory representations can equally be affected by the nature of the task, and studies have shown a clear link between where we look and what we recall (see e.g., Hollingworth and Henderson, 2002). Since more fixations are made to those items which are task-relevant, then it follows that it is these task-relevant items that will be best recalled.

However, more recent work has suggested that such advantages for the recall of task-relevant items are due to more than just increased fixations. Instead, the specific nature of the task can influence the way in which information is extracted from the objects and stored in memory. In one such study, it was found that searching for a target object was only benefitted by the object having been fixated during an earlier search for it. If the object had been fixated in the course of an earlier memory task where it was not the target, then no benefit for subsequent search was found (Võ \& Wolfe, 2012). Similarly, Tatler and Tatler (2013) showed that fixation number alone could not account for differences in memory. When the fixation number and memory performances were compared across task-relevant and irrelevant objects, it was found that for a given number of fixations, memory for task-relevant objects was significantly better, indicating that the task constraints had an influence on how much information was extracted during these fixations.

Within memory, it is not merely the object as a whole that is prioritized by its task relevance, but its separate properties and features too. Triesch, Ballard, Hayhoe, and Sullivan (2003) found that participants would retain property information across different stages of a task if that property was known to be necessary for future actions. More recently, evidence for object property prioritization has been shown in a study using the everyday task of tea-making (Tatler, Hirose, Finnegan, Pievilainen, Kirtley, and Kennedy, 2013). Participants who actively used the objects in the course of the study showed better spatial memory for the relevant items, and indications that they were de-prioritizing color information for these items. In contrast, control participants, who watched point-of-view videos of the task, but did not themselves interact with any of the objects, showed no such changes or evidence of prioritizing object features in their subsequent memory performance.
Based on this earlier work, it seems clear that in the process of a task, vision performs an active role, seeking out the items required to achieve the goal. Furthermore, the preparation and performance of action can influence the perception of objects in another way, by biasing attention in favor of particular object properties that are compatible with the nature of the action being performed. Such an effect is apparently strong enough to persist into the resulting memory representation, even after the completion of the task.

However, previous work has only used two groups of objects within tasks: those that are task-relevant, and therefore interacted with, and those which are not relevant, and therefore receive no interaction. As a result, there is a potential confound between the influence of task-driven interaction, and physical interaction with the objects which is not driven by the demands of the task. It is possible that the act of manipulation itself can also influence the way in which the representation is formed, and may do so separately from the influence of task-relevance. Thus, it is necessary to tease these two factors apart, in order to determine if they do have separate influences on the resulting object memory.

The aim of the present study, therefore, was to tease apart the contributions of task relevance and simple manipulation which does not serve the task. We examined the influence that each has on perception and inspection behavior, measured by the participant's eye movements, and memory, measured by recall performance on the various properties of the objects used.

In order to tease apart the contribution of these two factors, we required participants to interact with objects in two different ways: using an object in the course of completing a task (target), or moving an object out of the way (obstructing, but interacted with). Merely moving an object can be regarded as task-relevant itself (particularly if participants receive specific instructions to move the obstructing object in order to reach a target item). However, there is a clear difference between the two actions: the obstructing object will not be used for its usual purpose. In contrast, the target item has to be manipulated in order to achieve a particular stage of a task. For example, when making tea, a kettle is a specifically necessary object in order to start the task by boiling water. In contrast, any object obstructing access to the kettle (e.g., a plate or bowl) will not be used in its expected way, it will simply be moved. Thus, we feel there is enough of a distinction between the two action types for effects to be expected if task relevance itself influences inspection of and memory for objects.

Our findings will allow us to determine whether task relevance and physical manipulation of objects impart distinct influences on the inspection and representation of objects. If this is the case, such a finding would indicate that the nature of the task (and the participants' understanding of the goal) contributes to the manner in which objects are inspected and their representations are formed, which does not occur when simply picking up an object and cannot be accounted for by merely interacting with 
an object. Alternatively, it may be that there is no difference between the two types of interaction; in which case, any reason for interacting with an object will lead to similar effects on inspection and recall. This would also suggest that task relevance may not be the predominant factor, if simply holding an item is enough to prioritize it.

\section{Method}

\section{Participants}

Twenty participants (six males) with a mean age of 21.8 years (standard deviation $(\mathrm{SD})=1.63$ ) were recruited from the undergraduate population at the University of Dundee. Participants received course credits in return for their participation. All participants had normal vision without correction by glasses or contacts.

\section{Stimuli and apparatus}

Eye movement recording Participants wore the Positive Science LLC mobile eye tracker during the task. This tracker consists of two video cameras, mounted on the frame of a pair of glasses, both recording at $30 \mathrm{~Hz}$. One camera (mounted above the eye) faces forward to record the head-centered view of the scene, while a second camera (mounted below the eye) faces toward the participant, to record the movements of the right eye. The movies from each camera are recorded separately, and synchronized after the on-line recording into a single video, using the Yarbus software package (v. 2.2.3). Gaze direction was estimated via pupil tracking, and a nine-point calibration procedure at the start and end of the task. The system is accurate enough to allow spatial estimates of gaze direction to within a degree of visual angle.

Task setting Twenty-one objects were obtained and placed in a kitchen environment (Fig. 1a shows the layout of this environment). Within the object set, seven were necessary for a tea-making task (kettle, teapot, mug, spoon, milk jug, tea-bag box, sweetener container), seven for a task in which participants made a sandwich and poured a glass of juice (plate, bread-bin, peanut butter jar, jam jar, knife, juice bottle, glass), and the remaining seven were items compatible with a kitchen environment, but not themselves linked by a task set (toaster, cooking pot, salt cellar, bowl, fork, washing-up liquid, measuring jug). These objects acted as a control group, as they were items that were simply present in the scene, but not interacted with in any way.

In placing the items in the kitchen, the items were positioned in groups of three. These groups contained a tea-making object, a sandwich-making item, and a background item (e.g., kettle, plate, and toaster). Depending on the task that would be carried out, the relevant item would be obstructed by the alternative task item. For example, in the tea-making condition, the target kettle would be blocked by the obstructing plate, which was placed on the top of the kettle (Fig. 1b, left). In the sandwich-making task, the nowrelevant plate would be blocked by placing the obstructing kettle on top (Fig. 1b, right). Thus, in order to reach the obstructed target object, participants would have to interact with the obstructing object, by moving it out of the way. In both situations, the background item (in this example, the toaster) would be placed in the vicinity of the task pair, but would not hinder access to either of the items.

Stimulus questionnaire For each of the 21 objects, three questions were created to test participants' memories for the color, shape, and starting position of each of the objects. All question types were 4AFC questions. For color questions, the options were presented as color names, with each of the foil options plausible colors for the item. The shape options were presented as line drawings of possible examples of the object type. For the position questions, a line drawing of the environment was presented, with four locations marked with letter options. The foils in each case were all positions for another item in the kitchen. Examples of these three question types are shown in Fig. 2. The order in which the objects were asked about was randomized, as was the order of the property questions for each item.

Question presentation and response collection was controlled by scripts written in MatLab, using the PsychToolBox extensions (Brainard, 1997; Pelli, 1997). The question screens were displayed to the participants on the display monitor of a Macintosh computer, at a resolution of 1,024 $\times 768$.

\section{Procedure}

Participants were informed that they would carry out either a tea-making (half of the participants) or sandwich-making task. They were also told that there would be irrelevant items blocking the objects they required to successfully complete the task. In order to deal with these, they were told to move them to another table in the kitchen, located on the other side of the room. Participants were further instructed to move the blocking objects as they came to them at the stages of the task, rather than moving all items at the beginning of the task, as this prevented participants from moving all the blocking objects at the beginning of the experiment, and so avoided the possibility of primacy effects in memory for these objects. Participants were fitted with the mobile eye tracker and calibrated, then began the task. No time restriction was given but for both tea- and sandwichmaking the average time taken was around $3 \mathrm{~min}$. At end of the task, the tracker was removed, and participants were informed they would also perform a memory task for the items in the kitchen, of which they were previously unaware. The questions were presented on the laboratory computer, and participants 
a

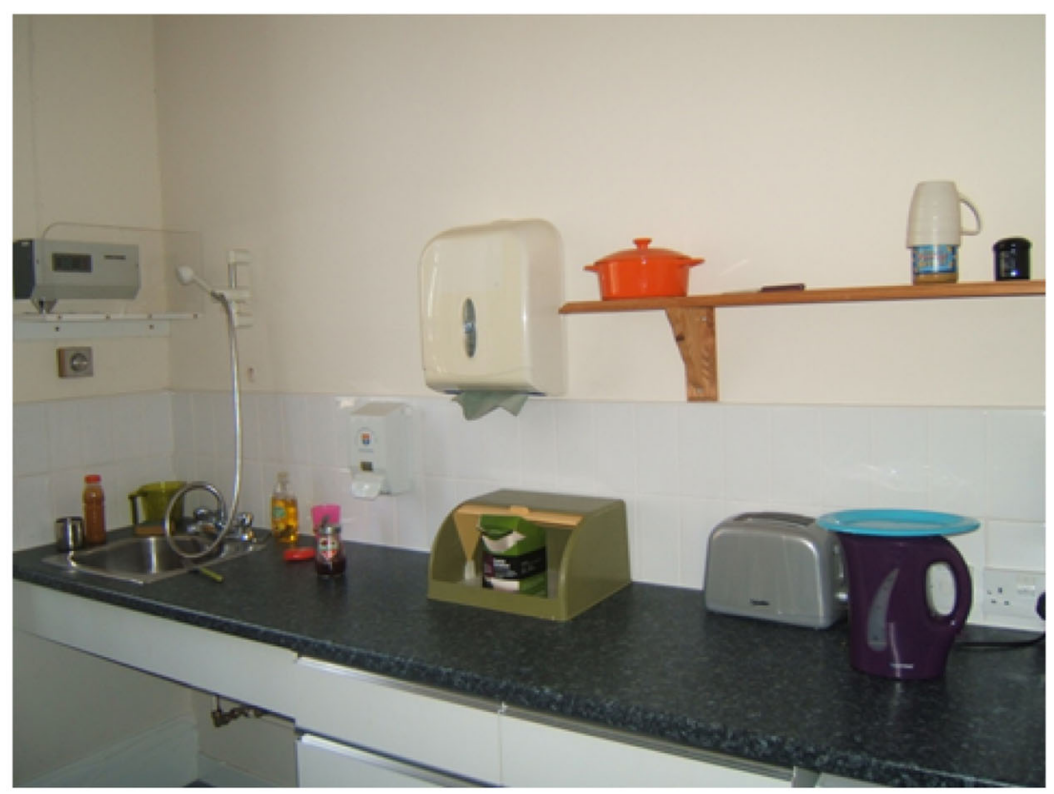

b

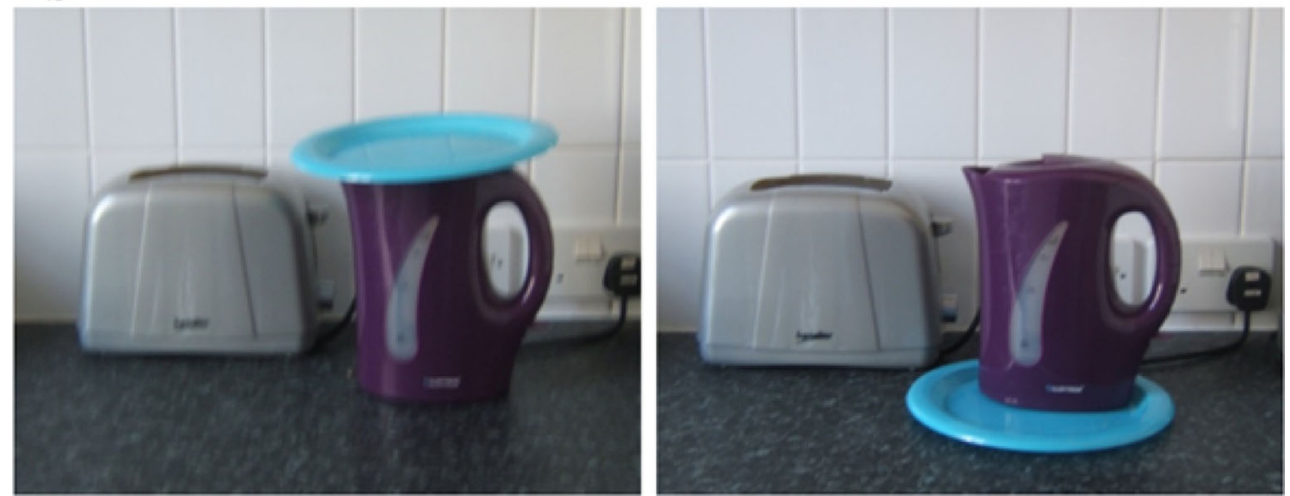

Fig. 1 (a) Photograph of the kitchen area and objects for the tasks; (b) examples of the object pair positions used; left: tea-making task; right: sandwichmaking task

responded using the keyboard, and the number keys 1-4, which had been relabelled A-D.

\section{Analysis}

The videos from the scene and eye cameras were combined to produce a single video for each participant, showing the gaze estimation for each frame of the movie. To analyse the data, manual frame-by-frame coding of the videos was conducted, by counting the number of frames in which each of the 21 objects was fixated, and the number of frames in which the necessary objects were gripped. Grip times were estimated via the scene camera as the times when the hands were visibly in contact with the objects.

Analysis of the effect of the types of interaction was performed using linear mixed effects models, using the lme4 package in the $\mathrm{R}$ statistical programming environment (Bates, Maechler, and Bolker, 2013; R Core Team, 2013).
This statistical approach offers considerable advantages over traditional analyses of variance approaches for the dataset and questions of the current study. LMMs are particularly well suited to datasets such as those collected in this study because they are able to deal with uneven distributions of data between conditions in the design, they can combine continuous and categorical factors within the same model, and they can measure variance across subjects and items simultaneously (Kliegl et al. 2012). Given the known link between fixation time and memory (e.g., Hollingworth \& Henderson, 2002), any account of effects of task or manipulation of objects on memory must take into account any differences that these factors have on fixation times. That is, we might expect that our three categories of object would be looked at for differing amounts of time and this alone might result in differences in memory performance. However, we were interested in characterizing whether task and manipulation influence memory above and beyond that arising from any differences in fixation times on these classes of objects. LMMs 

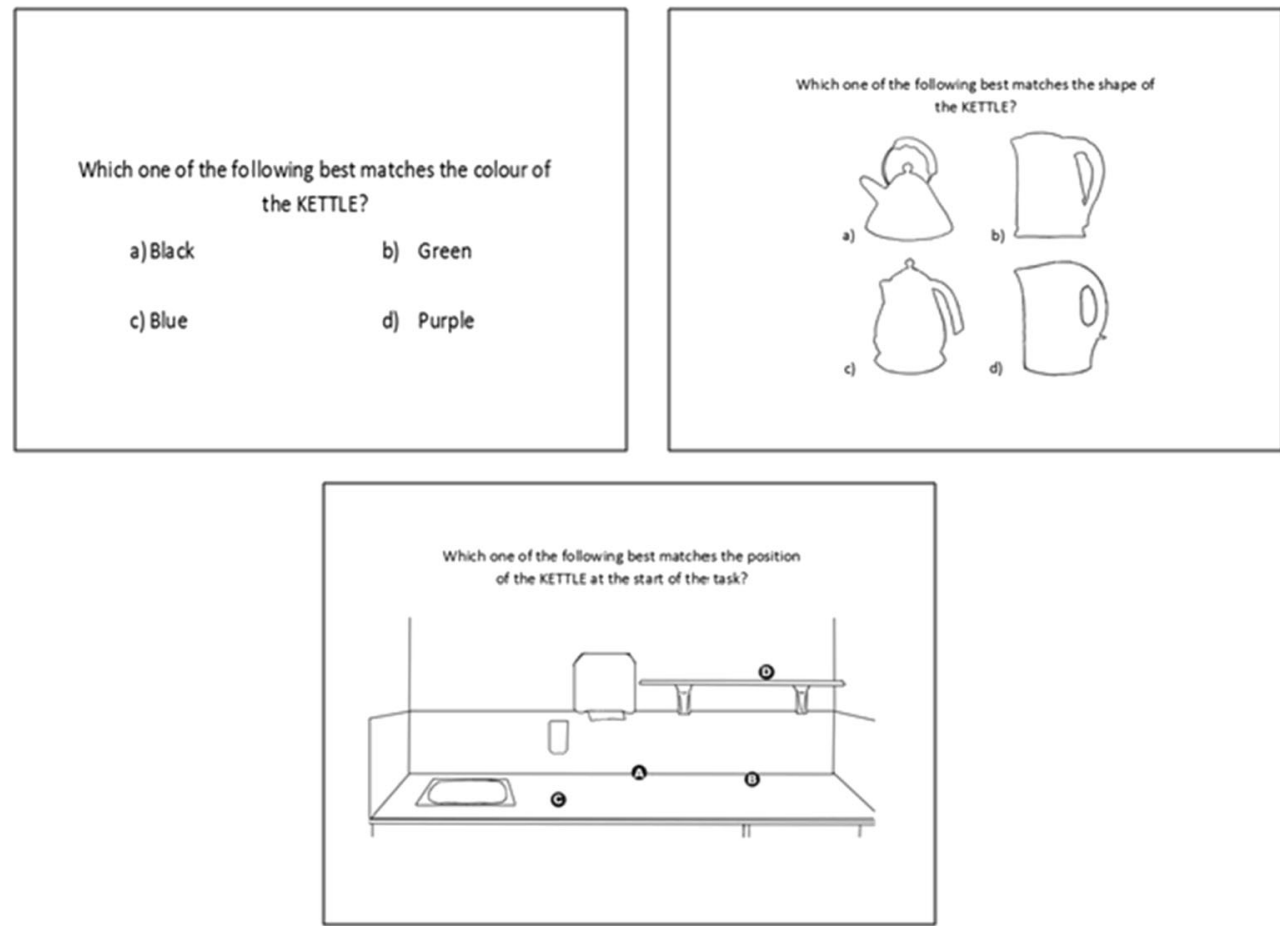

Fig. 2 Examples of the property questions: color, shape, and position

provide a statistical tool for asking this question. By including the continuous predictor of fixation time alongside the categorical predictor of object type, we can consider whether there are effects of task and manipulation that persist when effects of fixation time have been accounted for in the models. Thus any effects of task and manipulation in LMMs that include fixation time as a predictor will be effects above and beyond those arising from differences in fixation time. LMMs therefore offer the necessary statistical tools for addressing our theoretical aims.

Models were run to predict total fixation time (the summed duration of all fixations on the object), the total grip time (the summed duration of all grips on an object), and memory performance for each of the three property questions. The distributions of both the total fixation time and total grip time were examined, and subsequently log-transformed, in order to meet the assumptions of LMMs.

For modelling the total time spent either fixating or gripping the objects, the object type (target, obstructing, background) was used as a fixed factor, while subjects and objects were included as random factors. For modelling performance in the memory questions, logistic models were run using the object type (target, obstructing, background) and fixation time as the fixed factors, and subjects and objects as random factors. In all models, we used the most complex random effects structure that resulted in models that converged: that is we used models with random slopes and intercepts where possible (as recommended in Barr, Levy, Scheepers, and Tily, 2013). However, these maximal models often do not converge and in such cases we simplified stepwise by first removing the calculation of correlation parameters, followed by removing the random slope(s) for any interaction term(s), followed by removing random slopes for main effects. In all cases the results are reported from the model with the most complicated random effects structure that converged.

Both binomial and continuous models were run on the data, as appropriate. For the continuous models, the criterion for significance used was that the absolute $t$ values must be greater than 2. This is in agreement with earlier studies using LMMs (see e.g., Kliegl, Masson, and Richter, 2010).

\section{Results}

Objects that received no fixations during the task were excluded from the dataset prior to analysis, as we were interested in how information was extracted from fixations made to the objects under the different action conditions.

\section{Tasks $^{1}$}

To determine if participants in the tea- and sandwich-making groups acted similarly with the relevant objects, the task type

\footnotetext{
${ }^{1}$ In order to ensure that the three object sets (tea-making, sandwich-making, and background) were equally memorable, a set of nine participants memorised the objects in the study environment without any interaction. No significant difference was found between the three sets for any object property, indicating that all items in the three groups were equivalent in terms of memorability.
} 
(tea- or sandwich-making) was used to predict performance on the property questions and fixation behavior. Table 1 summarizes the results from these analyses on each of the three question types.

The results show that no difference was found between the two task groups for recall of any property. Similarly, the participants' group did not predict a significant difference in the total fixation time spent on objects, $\beta=0.143, \mathrm{SE}=0.138, \mathrm{t}=1.04$. Thus in the analyses that follow, data are collapsed across the two tasks.

Selection: fixation time Target items were fixated for significantly longer than background items, $\beta=1.23, \mathrm{SE}=0.088, \mathrm{t}=$ 13.96, as were the obstructing items, $\beta=0.574, \mathrm{SE}=0.087, \mathrm{t}=$ 6.63. The target items were also fixated for longer than the obstructing objects, $\beta=-0.659$., $\mathrm{SE}=0.085, \mathrm{t}=-7.72$. Figure 3 a shows the log-transformed fixation times to the three object types.

Selection: grip time In examining the total time spent gripping the objects, the items in the background condition were removed from analysis, as these items were never interacted with. Participants held the target items for significantly longer than obstructing items, $\beta=-0.598, \mathrm{SE}=0.036, \mathrm{t}=-16.57$. Figure $3 \mathrm{~b}$ displays the log-transformed grip times for the two object groups.

Memory task Performance on object memory was examined for each of the three property questions using an LMM model which incorporated both object type and fixation time as fixed effects, and subjects and objects as random effects. This allowed us to consider the influence of the object type on memory performance above and beyond those resulting from differences in fixation time. Figure 4 displays the proportion of correct responses for the three property questions, across the three object types (note that unlike the models that follow, these plots necessarily do not account for differences in performance attributable to differences in fixation time).

\section{Position}

Position information was unaffected by the time spent fixating the objects, $\beta=0.484, \mathrm{SE}=0.350, \mathrm{z}=1.38, \mathrm{p}=0.166$. The task

Table 1 Summary of linear mixed effects analyses comparing memory performance for the three object properties between participants in teamaking and sandwich-making tasks

\begin{tabular}{llll}
\hline & $\beta$ & SE & $\mathrm{z}$ \\
\hline Position & -0.209 & 0.260 & -0.803 \\
Color & -0.391 & 0.380 & -1.03 \\
Shape & -0.275 & 0.323 & -0.851 \\
\hline
\end{tabular}

did not significantly influence memory for this property, as no significant difference was found between the target $(M=0.66)$ and obstructing items $(M=0.58), \beta=-0.129, \mathrm{SE}=0.441, \mathrm{z}=$ $-0.292, p=0.770$. An influence of manipulation was similarly absent, with no significant difference seen between obstructing and background objects, $\beta=0.076, \mathrm{SE}=0.571, \mathrm{z}=0.133, \mathrm{p}=$ 0.894 . Furthermore, no significant difference was found between the recall of the positions of target and background objects $(M=$ $0.52), \beta=0.173, \mathrm{SE}=0.706, \mathrm{z}=0.245, \mathrm{p}=0.806$.

\section{Color}

For color questions, performance was not influenced by the total fixation time, $\beta=0.320, \mathrm{SE}=0.328, \mathrm{z}=0.974, \mathrm{p}=0.330$. Object type was a significant predictor of performance, demonstrating the importance of task influence, as the color of target items $(M=$ $0.65)$ was recalled significantly better than background items, $(M$ $=0.26), \beta=1.55, \mathrm{SE}=0.658, \mathrm{z}=-2.36, \mathrm{p}=0.018$. However, no difference was found between recall for obstructing items $(M=$ 0.45 ) and target items, $\beta=-0.750, \mathrm{SE}=0.472, \mathrm{z}=-1.59, \mathrm{p}=$ 0.112 , nor between obstructing and background items, $\beta=$ $-0.801, \mathrm{SE}=0.498, \mathrm{z}=1.61, \mathrm{p}=0.108$, indicating that there was no strong effect of manipulation for this property.

\section{Shape}

Recall of shape information was not influenced by the total fixation time, $\beta=-0.184, \mathrm{SE}=0.463, \mathrm{z}=-0.397, \mathrm{p}=0.691$. There was an effect of task, with participants displaying significantly better recall of the shape of target items $(M=0.74)$ compared to obstructing items $(M=0.58), \beta=-1.30, \mathrm{SE}=$ $0.558, \mathrm{z}=-2.33, \mathrm{p}=.020$. The manipulation of objects also had an effect, as participants were significantly better at recalling both the shape of obstructing items compared to background items, $\beta=1.53, \mathrm{SE}=0.479, \mathrm{z}=3.20, \mathrm{p}=$ 0.001 , and the target objects compared to background items $(M=0.28), \beta=2.82, \mathrm{SE}=0.802, \mathrm{z}=3.51, \mathrm{p}<.001$.

It was possible that the time participants spent gripping the objects could also influence the memory performance, particularly the recall of shape. Grip time was included as a fixed factor, along with object type. As for the previous grip analyses, the background objects (which were never interacted with) were removed from this part of the analysis. With both grip time and object type included, grip time was not a significant predictor of color, $\beta=0.181, \mathrm{SE}=0.093, \mathrm{t}=-1.96$; shape, $\beta=-0.074, \mathrm{SE}=0.089, \mathrm{t}=-0.834$, or position recall, $\beta=-0.088, \mathrm{SE}=0.087, \mathrm{z}=-1.01$.

\section{Discussion}

The present study examined the influences of task-driven and simple physical interaction on inspection and memory. 
Fig. 3 Log-transformed total fixation times for target, obstructing, and background objects (left) and log-transformed total grip times for target and obstructing (right). Error bars shown represent standard error

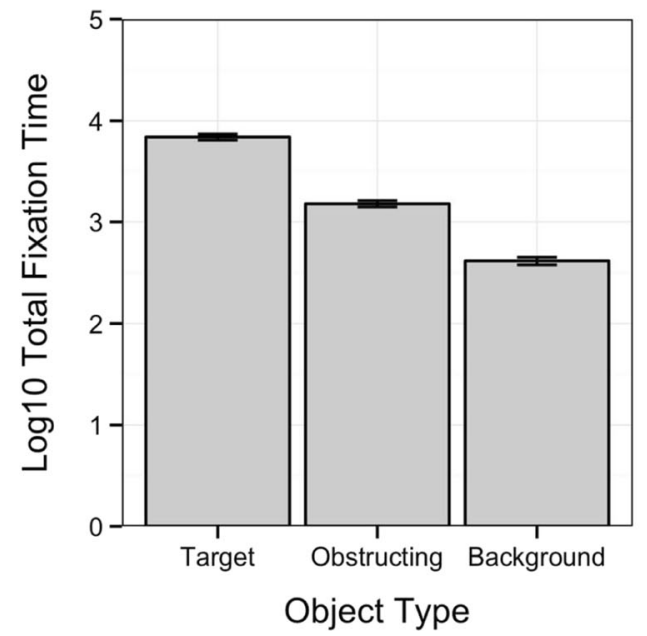

Participants spent the most time fixating target objects, and recalled their properties more accurately than those objects
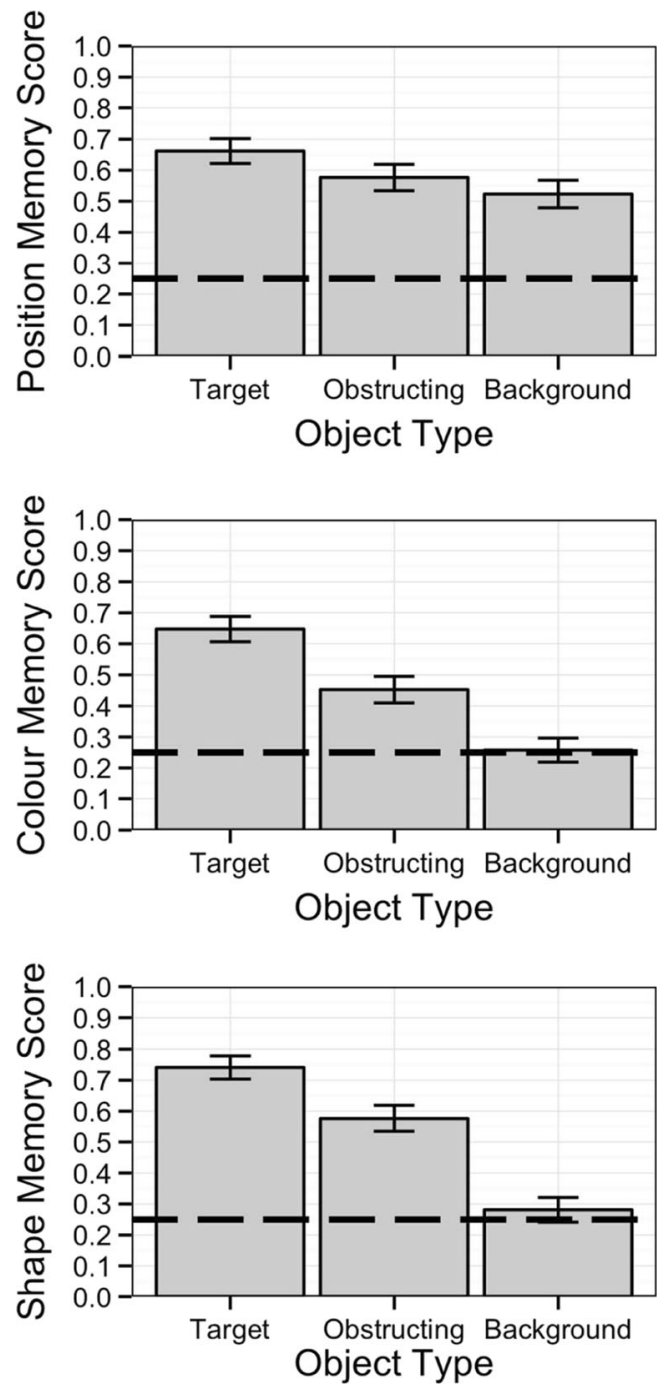

Fig. 4 Proportion of active participants' correct responses to target, obstructing, and background objects for color, shape, and position questions. Error bars shown represent standard error that had not been used. However, it emerged that simple interaction with obstructing items also influenced both the inspection and recall of these objects compared to unused items, with participants showing longer fixation times and better recall of task-irrelevant items compared to the background objects. Differences in memory and fixation patterns still emerged between target object use and simple interaction, indicating that the use of objects in order to achieve a task goal has an influence beyond that derived from merely holding the objects, and the two types of interaction do have separate effects, although these differ for different properties.

\section{Selection: fixation and grip time}

The fixation patterns in the current study are much what would be expected based on previous research. Participants spend longer looking at items that are relevant to their task, replicating a well-established finding from both laboratorybased (e.g., Yarbus, 1967) and real-world situations (e.g., Land et al., 1999). Increased fixation time on such items is clearly necessary, allowing participants to gain the information they need to successfully work with the items, and complete the task.

We found a difference in fixation times between obstructing items, and the other two object types. While obstructing items were not fixated for as long as the target objects, they were still attended to for longer than the background objects. Clearly, the obstructing items were not required for as long within the task setting; participants simply need to locate and then move them, rather than having to obtain visual feedback during more complex manipulations. However, they did still require some interaction, and so the time spent fixating them was increased. This is in line with previous work demonstrating the close link between task and fixation: participants follow a "do it where I'm looking" strategy, attending to those objects that are required for the current stage of a task (e.g., Ballard et al., 1992). Thus, these 
differences in fixation time across the object groups are as expected, given the different requirements for using the objects.

A similar finding is evident for the differences in grip time across the two object groups that were interacted with. The target objects were those which were gripped for the longest total time period throughout the task, as they were the objects which require the more detailed manipulation to successfully complete the task. In contrast, the obstructing items simply needed to be moved out of the way, a much simpler requirement.

However, when considering the influence either of these factors, fixation and grip time, on recall performance, it appears that neither of these factors had a strong influence on memory. The relationship between the object and the task was a better predictor of performance, beyond the effects from fixation or grip time and revealed separate influences of both task and manipulation on memory for object properties.

\section{Influence of task on object property recall}

The present study shows that the object properties (with the exception of position memory) are strongly influenced by the relevance of the object in a manner that cannot be accounted for merely by differences in fixation time. In order to consider these effects more closely, we examined recall performance for the three properties tested, across the three types of object.

Position Recall of position information was unaffected by the task demands. Participants showed high performances recalling the position of all items, regardless of which object set they belonged to. This is in contrast to the previous finding of Tatler et al. (2013), in which position information was prioritized for the task-relevant items. One possibility for the current study's finding of good position memory for all objects irrelevant of task status is the way in which the study itself was structured. As noted, objects were placed in groups of three (target, obstructing, and background), so that the obstructing objects blocked the target items, and the background objects were nearby. As a result, the AFC questions used at the recall stage show very similar positions for target and obstructing items in particular. Participants may therefore have used this to answer the questions: if the position of target items was maintained, they would only have to recall the identity of the object that blocked it, and then use the position of the target item to infer the positions of the irrelevant and background items. Thus, position recall would be generally high for all object groups, as is seen in our results. This possibility suggests that the results for position memory are not as reliable as those for color and shape. Future research in this area might benefit from using a different method of measuring position (e.g., memory for relative positions between objects). However, the findings for color and shape memory could not be affected by the experimental set-up in this manner, thus we suggest that these results do indicate that property memory may be differently affected by the relevance of the object to the task, and incidental manipulation.

Color Task relevance was a significant predictor of performance for color, with participants significantly better at recalling the colors of the objects necessary for the task than background objects' colors. However, no difference was found between color recall for obstructing and background objects, or obstructing and target items.

Previous studies which have used the differences between object properties (see e.g., Tipper, Paul, and Hayes, 2006) have considered color to be a strongly visual property, and as such, of not much relevance to actions conducted with objects. In this respect, the lack of a difference between some of the conditions is what might be expected: color information is not important to the task, and so is not prioritized. Thus, the advantage shown for recalling the target object colors over background object colors is unexpected. One possible explanation is that color is prioritized in order to aid the recognition of objects. Unlike the background objects, where the identity is unimportant, the target item is relevant precisely because of what it is, and the role it plays in achieving the end goal. As a result, color information may be extracted and maintained as a way of helping to identify the relevant objects.

However, this explanation would suggest that we should also see a difference between the recall of color for target and obstructing items. The identity of the obstructing items is similarly unimportant for the performance of the overall task, so if it is the case that color information is being used to aid identification, memory for the purely manipulated items would be worse than that for the target objects. This difference did not emerge.

Shape The most important results for this study were found in the results for the property of shape. Participants were significantly better at recalling the shapes of objects necessary for the task compared to both the obstructing and background objects. A significant difference was also found between obstructing and background items, with the shape of the manipulated items remembered more accurately than those that were never interacted with.

The findings for this property are in accordance with the nature of the property, which is considered to be strongly action-relevant (although it can also be seen as having visual aspects, since the shape and appearance of an object are practically indistinguishable, see e.g., Jeannerod, 1994). The shape of an item informs the grip that must be formed in order to interact successfully with it: both target and obstructing items must be interacted with, and so prioritization of this information is important, more so than for the objects in the background group. 
However, the most important and unique aspect of this finding for the present study is the fact that there was a difference in memory between the target and obstructing object groups. We suggest that this difference may indicate the different way objects in the two conditions are used. As suggested by Jeannerod (1994), an item's shape is a very clear indication of what it is, and how it should be used for a task, and so whether it is suitable to be used for a particular stage in achieving an end goal. This is not the case for obstructing objects: shape only informs the actions required to move the object, whatever it happens to be. Thus, while shape information is important for both object types, it is a different quality of importance, and so it seems that the prioritization of this information in memory differs for obstructing and target items. Using an object as part of a task to achieve a goal therefore seems to influence the construction of a representation beyond changes resulting from the simple manipulation of the objects.

\section{Task and manipulation in object inspection and represen-}

tation We have found further evidence to show that the nature of a task influences not only where participants look during the task, but also what information they best recall from the objects used.

The present study shows that the object properties (with the exception of position memory) are strongly influenced by the relevance of the object in a manner that cannot be accounted for merely by differences in fixation time. This adds further support to studies by Võ and Wolfe (2012), Tatler and Tatler (2013), and Tatler et al. (2013), which similarly indicate that the task relevance of an object contributes to memory performance beyond that accounted for by fixation time. Rather than the differences in fixation time influencing memory for these items (as suggested by Hollingworth, 2006, 2012), it seems there is a difference in the way in which information is extracted from these fixations. Task-relevance therefore appears to lead to strategic prioritization in the encoding and retention of information from such objects.

The current study extends previous understanding by demonstrating that simple physical interaction with the objects will lead to similar changes in fixation time and memory. These findings are in agreement with earlier work by Thomas, Davoli, and Brockmole (2013), which compared memory for objects that were either only viewed or picked up as well. Interaction with items was found to affect the recall of the spatial environments, with objects remembered as closer together, within a smaller area than physically presented. While there are differences between Thomas et al.'s (2013) findings and those of the present experiment (e.g., the current study shows an improvement in memory accuracy for properties, rather than a distortion in recall), both studies indicate that simply acting with an object is enough to change the memory formed.
However, our results show for the first time that physical interaction and actions driven by task goals do not lead to identical effects in memory: the use of an object as part of task instructions leads to further biasing of some object information (namely the object's shape), beyond the changes due to a task-irrelevant interaction.

Theories of grounded cognition (e.g., Barsalou, 2008) suggest a close link between action and perception, with each able to influence the other. Such an influence allows for preparation, from perception of an object cuing a suitable response to the intention to act prompting early selection of necessary objects. Recent studies, and the current findings, show that this situational weighting can also affect the construction of a memory representation, persisting after the task is completed. Furthermore, the present study suggests this weighting may be particularly sensitive to the task demands, with differently constructed representations depending on why the objects are manipulated in the course of the task.

Further research is required to investigate more real-world situations, and how the changing context might affect the weighting of properties in representations. Properties such as color and position may be particularly affected by the nature of the task, and studies in which the setting is purposefully manipulated to make these features explicitly relevant (or not) would reveal more about how the task demands influence the biased construction of memory. The finding that these effects persist in memory beyond the task conclusion also bears further investigation, into how long such weightings might persist, and their potential influence on subsequent tasks.

In conclusion, the current study demonstrates that, in realworld settings, the task is a significant factor in both directing our fixations and encoding the information in memory. Most importantly, we demonstrate for the first time that any physical interaction can lead to changes in viewing and memory strategies. Both the use of objects in the course of a task and incidental interaction with items leads to changes in the way information about relevant object features (in particular, the shape) is encoded and subsequently recalled.

Author note Portions of this research were presented at the Experimental Psychological Society conference at the University of Kent (May, 2014).The first author is supported by a studentship provided by the University of Dundee. This study was conducted as part of the requirements for the degree of Doctor of Philosophy by the first author.

\section{References}

Ballard, D. H., Hayhoe, M. M., Li, F., \& Whitehead, S. D. (1992). Handeye coordination during sequential tasks. Philosophical Transactions of the Royal Society of London, 337, 331-339.

Barr, D. J., Levy, R., Scheepers, C., \& Tily, H. J. (2013). Random effects structure for confirmatory hypothesis testing: Keep it maximal. Journal of Memory and Language, 68(3), 255-278. 
Barsalou, L. W. (2008). Grounded cognition. Annual Review of Psychology, 59, 617-645.

Bates, D. M., Maechler, M., \& Bolker, B. (2013). Ime4: Linear mixedeffects models using S4 classes (R package version 0.999999-2). http://CRAN.R-project.org/package=lme4

Brainard, D. H. (1997). The psychophysics toolbox. Spatial Vision, 10, 433-436.

Fagioli, S., Hommel, B., \& Schubotz, R. I. (2007). Intentional control of attention: Action planning primes action-related stimulus dimensions. Psychological Research, 71(1), 22-29.

Hayhoe, M. M., Shrivastava, A., Mruczek, R. E. B., \& Pelz, J. B. (2003). Visual memory and motor planning in a natural task. Journal of Vision, 3, 49-63.

Hollingworth, A. (2006). Visual memory for natural scenes: Evidence from change detection and visual search. Visual Cognition, 14(48), 781-807.

Hollingworth, A. (2012). Task specificity and the influence of memory on visual search: Comment on Võ and Wolfe (2012). Journal of Experimental Psychology: Human Perception and Performance, 38(6), 1596-1603.

Hollingworth, A., \& Henderson, J. M. (2002). Accurate visual memory for previously attended objects in natural scenes. Journal of Experimental Psychology: Human Perception and Performance, 28(1), 113-136.

Jeannerod, M. (1994). The representing brain: Neural correlates of motor intention and imagery. Behavioural Brain Sciences, 17, 187-245.

Kliegl, R., Dambacher, M., Dimigen, O., Jacobs, A. M., \& Sommer, W. (2012). Eye movements and brain electric potentials during reading. Psychological Research, 76(2).

Kliegl, R., Masson, M. E. J., \& Richter, E. M. (2010). A linear mixed model analysis of masked repetition priming. Visual Cognition, 18, 655-681.

Land, M. F., Mennie, N., \& Rusted, J. (1999). The roles of vision and eye movements in the control of activities of daily living. Perception, 28(11), 1311-1328.
Pelli, D. G. (1997). The VideoToolbox software for visual psychophysics: Transforming numbers into movies. Spatial Vision, 10, 437-442.

R Core Team. (2013). R: A language and environment for statistical computing. Vienna, Austria: R Foundation for Statistical Computing. URL: http://www.R-project.org/

Symes, E., Tucker, M., Ellis, R., Vainio, L., \& Ottoboni, G. (2008). Grasp preparation improves change detection for congruent objects. Journal of Experimental Psychology: Human Perception and Performance, 34(4), 854-871.

Tatler, B. W., Hirose, Y., Finnegan, S. K., Pievilainen, R., Kirtley, C., \& Kennedy, A. (2013). Priorities for selection and representation in natural tasks. Philosophical Transactions of the Royal Society B, 368(1628), 1-10.

Tatler, B. W., \& Tatler, S. L. (2013). The influence of instructions on object memory in a real- world setting. Journal of Vision, 13(2), $1-13$.

Thomas, L. E., Davoli, C. C., \& Brockmole, J. R. (2013). Interacting with objects compresses environmental representations in spatial memory. Psychonomic Bulletin \& Review, 20(1), 101-107.

Tipper, S. P., Paul, M. A., \& Hayes, A. E. (2006). Vision-for-action: The effects of object property discrimination and action state on affordance compatibility effects. Psychonomic Bulletin \& Review, 13(3), 493-498.

Triesch, J., Ballard, D. H., Hayhoe, M. M., \& Sullivan, B. T. (2003). What you see is what you need. Journal of Vision, 3, 86-94.

Võ, M. L.-H., \& Wolfe, J. M. (2012). When does repeated search in scenes involve memory? Looking at versus looking for objects in scenes. Journal of Experimental Psychology. Human Perception and Performance, 38(1), 23-41.

Wilson, M. (2002). Six views of embodied cognition. Psychonomic Bulletin \& Review, 9(4), 625-636.

Yarbus, A. L. (1967). Eye movements and vision. New York: Plenum Press. 